\title{
How to Deal with an Intraoperative Thrombosis of Microvascular Anastomosis
}

\author{
Lawrence Van Look1, Tomas Menovsky ${ }^{1,2}$, Gino Vissers ${ }^{1,3}$, Thierry Tondu, ${ }^{1,3}$, Filip Thiessen ${ }^{1,3}$ \\ ${ }^{1}$ Faculty of Medicine and Health Sciences, Antwerp University, Edegem, Belgium \\ ${ }^{2}$ Department of Neurosurgery, Antwerp University Hospital, Edegem, Belgium \\ ${ }^{3}$ Department of Plastic Surgery, Antwerp University Hospital, Edegem, Belgium \\ Email: lawrence.vanlook@student.uantwerpen.be
}

How to cite this paper: Van Look, L., Menovsky, T., Vissers, G., Tondu, T. and Thiessen, F. (2021) How to Deal with an Intraoperative Thrombosis of Microvascular Anastomosis. Open Journal of Modern Neurosurgery, 11, 281-295.

https://doi.org/10.4236/ojmn.2021.114033

Received: September 21, 2021

Accepted: October 26, 2021

Published: October 29, 2021

Copyright $\odot 2021$ by author(s) and Scientific Research Publishing Inc. This work is licensed under the Creative Commons Attribution International License (CC BY 4.0).

http://creativecommons.org/licenses/by/4.0/

\begin{abstract}
Background: Intraoperative thrombosis during microvascular surgery is a nasty complication. Most intraoperative thromboses occur at the proximity of the anastomosis and microsurgical salvage techniques are needed to correct the complication. The aim of this article is to provide an overview of basic clinical patency testing and microsurgical salvage techniques. Methods: A search of the literature up to November 2020 was performed, using PubMed and Web of Science databases. Articles reporting on clinical intraoperative patency testing and/or salvage techniques in microvascular surgery were included. Results: Comprehensive illustrations of intraoperative clinical patency testing include: pulsation pattern, flicker test and milking test. The following surgical salvage techniques for both end-to-end and end-to-side intraoperative microvascular occlusion management are described: suture-line thrombectomy, thrombectomy through arteriotomy, anastomotic resection with complete re-anastomosis and, balloon extraction. Conclusion: Decision making in surgical salvage techniques for microvascular thrombosis depends on localization of the thrombus and the surgeon's experience and preference. In case of any doubt, it is better to reopen a few sutures and have a clear inspection of the anastomosis in order to prevent redo surgeries. This paper serves as a guide for especially the starting microsurgeon to clinically and surgically identify and handle an intraoperative microvascular anastomosis thrombosis and occlusion.
\end{abstract}

\section{Keywords}

Microvascular, Intraoperative, Thrombosis, Patency, Salvage, Complication 


\section{Introduction}

Intraoperative thrombosis during microvascular surgery is a nasty complication. Failure rate in free flap surgery is reported between $2 \%$ and $15 \%$ mostly due to thrombosis [1] [2] [3] [4] [5]. Risk factors for intraoperative thrombosis are: technical errors during microvascular surgery (endothelium manipulation and damage), significant vessel size mismatch, poor vessel quality and smoking [6] [7] [8]. Patency of the anastomosis is examined by various methods: clinical examination, Doppler, indocyanine green video-angiography and infrared thermography. As not every hospital has this technology, we tend to provide a set of tools for patency testing and salvage techniques that can be used without any sophisticated equipment. Therefore the clinical and surgical techniques, for both patency testing and salvaging, are the focus of this article. When an intraoperative thrombosis occurs, the clot is usually located at the anastomosis, and surgical salvage techniques are needed to manage the occlusion [3]. The aim of this article is to provide an overview of basic clinical patency testing and microsurgical salvage techniques for both end-to-end and end-to-side anastomosis.

\section{Materials and Methods}

\subsection{Data Sources}

The PubMed and Web of Science databases were searched up to November 2020. The following search terms were used: intraoperative, thrombosis, microvascular surgery, salvage techniques, patency, patency test and free flap. The selected articles were reviewed on title and abstract to determine study eligibility. Searches were supplemented by hand searching the reference lists of studies and through personal discussion. After reviewing the search through the databases, 11 articles were withheld. Two supplementary articles and one book were also used and cited.

\subsection{Study Eligibility}

Original articles fulfilling the following eligibility criteria are included: 1) the study involved a microvascular anastomosis; 2) the study evaluated intraoperative thrombosis; 3 ) any type of patency test and/or technical salvage procedure was performed; 4) the article was published in English, French, German or Dutch. The exclusion criteria were: a) studies that evaluated thromboses in vessels without anastomosis; b) studies that evaluated macrovascular thrombosis.

\section{Results}

\subsection{Surgical Patency Technique}

Intraoperative assessment of the anastomosis starts with the inspection of the vessel. After removal of the temporary clips, two types of pulsations of the artery distal to the anastomosis can be observed: expansional and longitudinal (see Figure 1(a)) [9]. Expansional pulsations enlarge an artery circumferentially and indicate a patent anastomosis. Longitudinal pulsations lengthen an artery and 


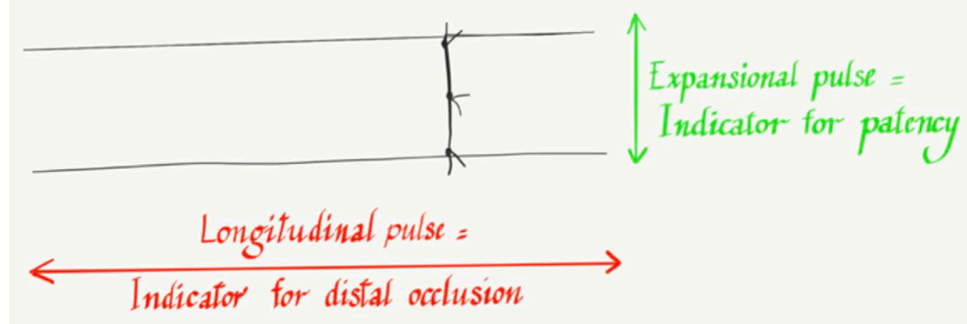

(a)

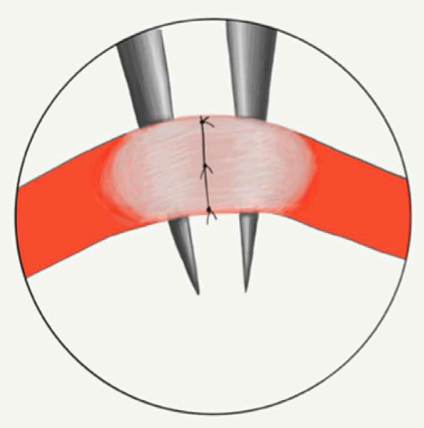

(b)

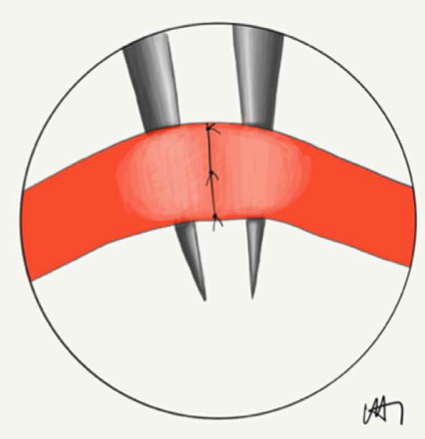

Figure 1. (a) Pulsation pattern; (b) Flicker test.

indicate a partially or completely occluded anastomosis. Besides absence of expansional pulsations, many early occluded anastomoses exhibit a white plug protrusion pushed through the suture-line. In other words, protrusion of a white plug is a strong indication of an occluded anastomosis [9].

If there is doubt about the patency of the microvascular anastomosis, the following test can be done. With the flicker test, closed forceps are placed underneath the anastomosis and with gentle upward pressure the artery becomes more narrow (see Figure 1(b)). During systole, blood flow through the narrowed patent anastomosis can be visualized through the thin and translucent arterial wall under the microscope [9]. In an occluded anastomosis, no flow of blood will be seen through the microscope. If the flicker test is inconclusive, the milking test can be performed. The milking test (also known as "Acland's test") involves milking the recipient artery with microforceps to empty and refill it with flow through the anastomosis (see Figure 2(a) \& Figure 2(b)) [9]. Two microforceps are held closely together to squeeze the artery distal to the anastomosis. The artery is stroked by sliding the distal forceps further distally to produce a blood free segment of several millimeters long. The proximal forceps are then reopened and blood fills the distal vessel through a patent anastomosis.

Venous microvascular thrombosis tends to manifest itself after the first 24 hours and is not often seen intraoperatively [10]. Therefore the salvage procedures will mostly be performed during a revision-surgery. Thrombosis of venous microvascular anastomosis is often due to two factors: i.e. lower blood flow 

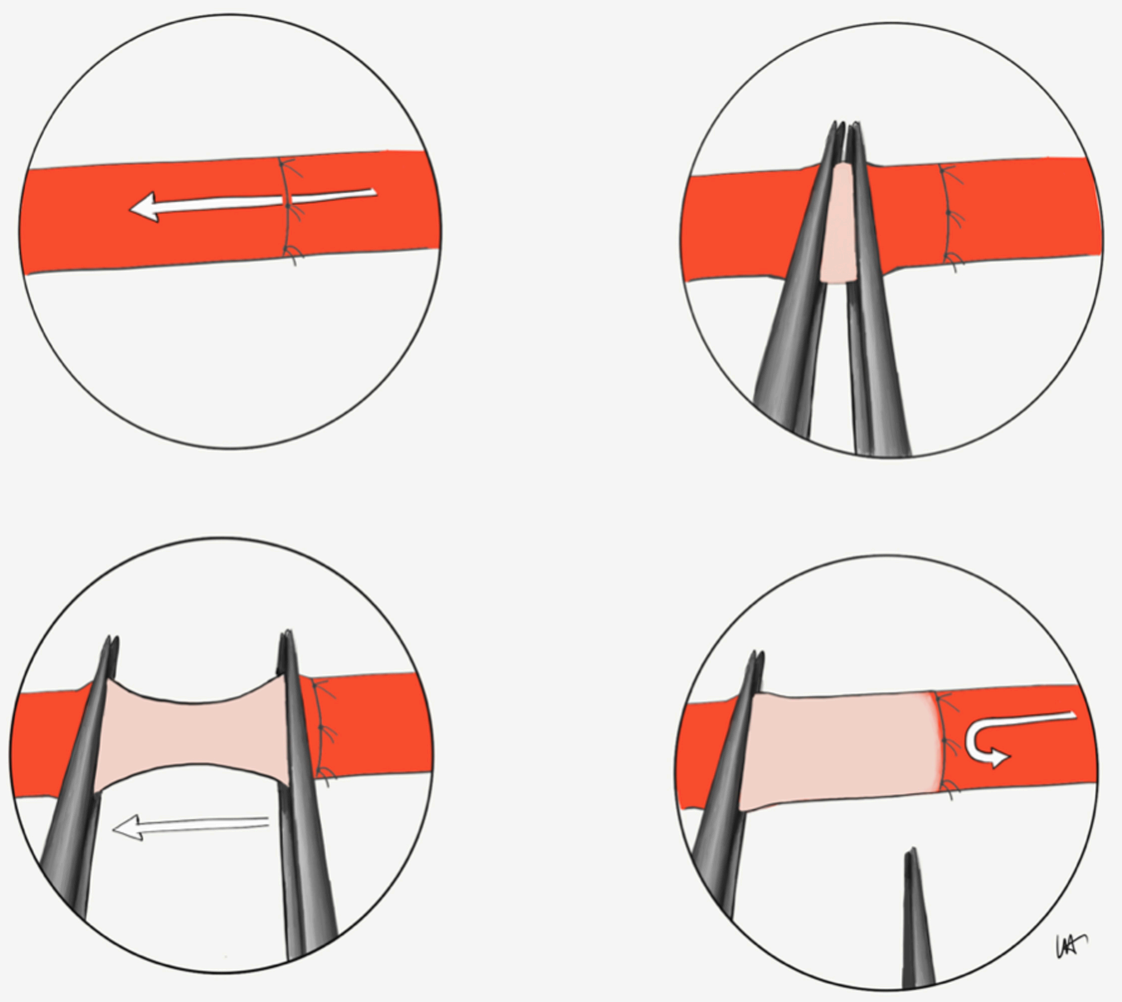

(a)
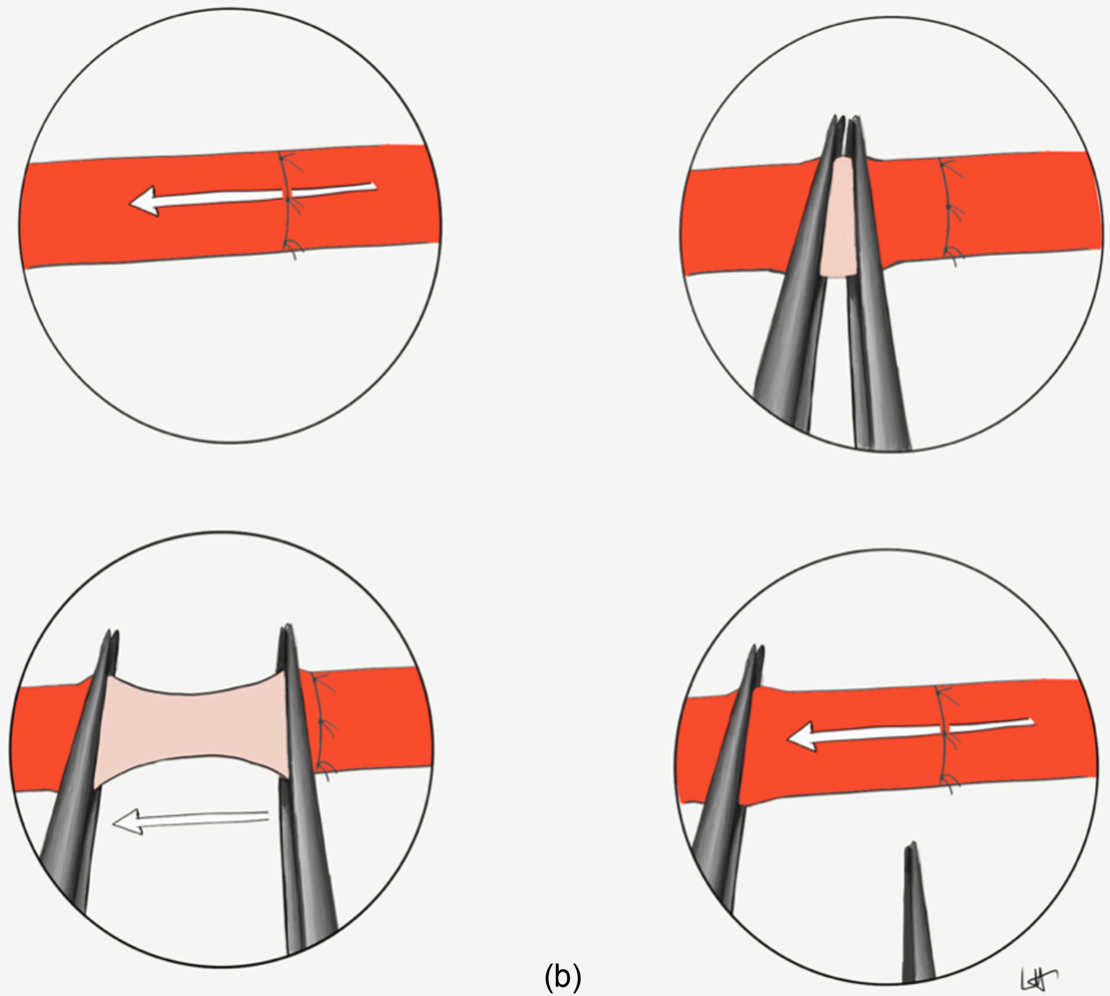

Figure 2. (a) Milking occlusion; (b) Milking patent. 
and more fragile and thinner vein wall with an increased possibility of iatrogenic damage during microvascular surgery. The previously mentioned patency tests are also used for venous anastomosis. To avoid iatrogenic endothelial damage, cautious tissue handling is extremely important in venous patency testing. The flicker test is less traumatic for the vein and therefore primarily preferred above the milking test.

\subsection{Surgical Salvage Technique: End-to-End Anastomosis}

\subsubsection{Suture-Line Thrombectomy}

The suture-line thrombectomy technique can be used for a small thrombus located at the anastomosis (see Figure 3) [9]. Two or three stitches of the anastomosis are removed to inspect the lumen and thrombus [3]. The thrombus is then

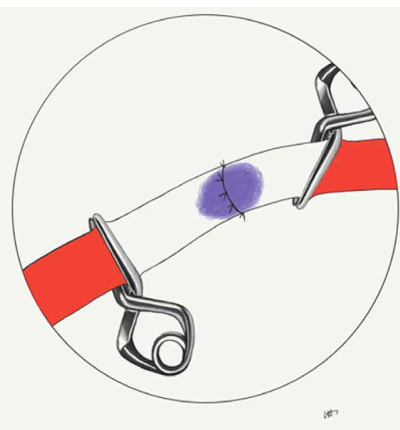

(a)

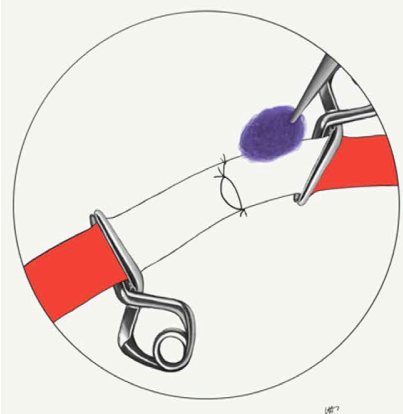

(c)

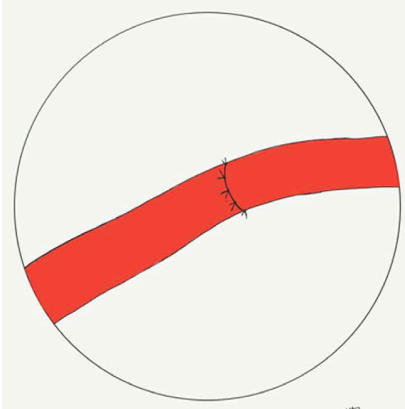

(e)

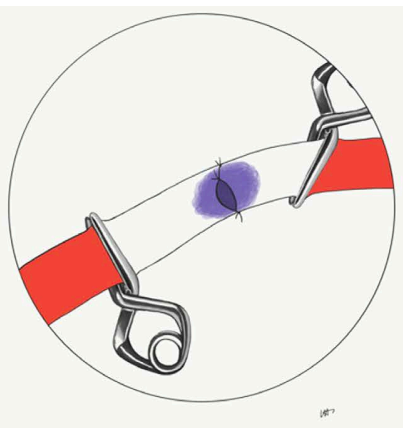

(b)

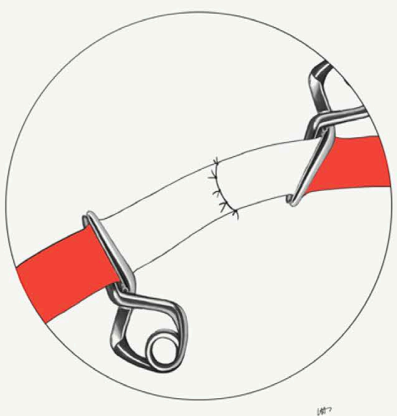

(d)

Figure 3. Suture-line thrombectomy. 
removed using the microforceps and hereafter the vessel ends are irrigated with a heparin solution.

\subsubsection{Thrombectomy through Arteriotomy}

Thrombus extraction can be achieved by a longitudinal arteriotomy of the vessel without dissembling the anastomosis. The arteriotomy is closed by a running suture after thrombus extraction [9].

\subsubsection{Resection of the Anastomosis with Complete Re-Anastomosis}

Resection of the whole anastomosis with complete re-anastomosis is recommended when suture-line thrombectomy is unsuccessful [3]. The anastomosis is resected at least $2-3 \mathrm{~mm}$ from each side of the thrombus, after which the donor and recipient vessels are reconnected (see Figure 4) [11].

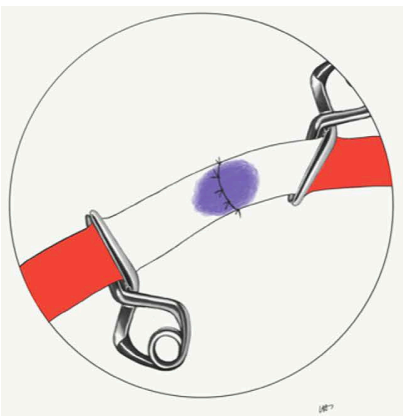

(a)

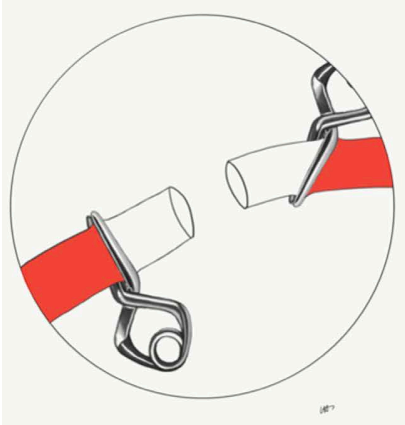

(c)

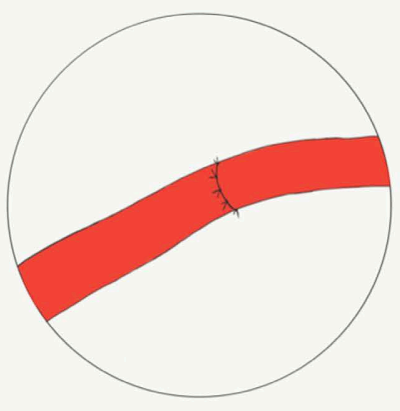

(e)

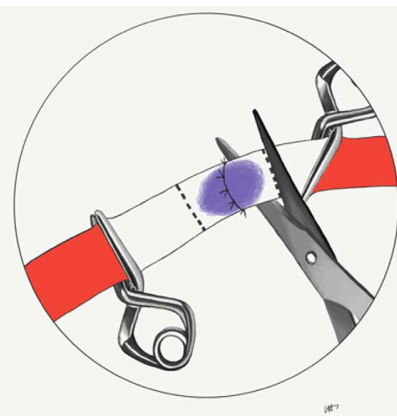

(b)

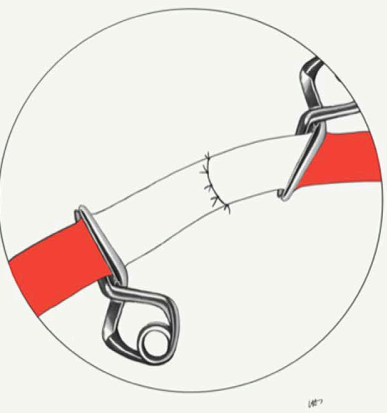

(d)

Figure 4. Complete re-anastomosis end-to-end. 


\subsection{Surgical Salvage Technique: End-to-Side Anastomosis}

\subsubsection{Suture-Line Thrombectomy}

The first option is, to reopen a few sutures at the suture-line, similar to Figure 3, and, to remove the thrombus.

\subsubsection{Thrombectomy through Arteriotomy}

Second option is a thrombectomy through a longitudinal arteriotomy at the donor artery (see Figure 5). With this technique, no sutures of the anastomosis are disrupted. After extraction of the thrombus, the arteriotomy is closed with a running suture [9].

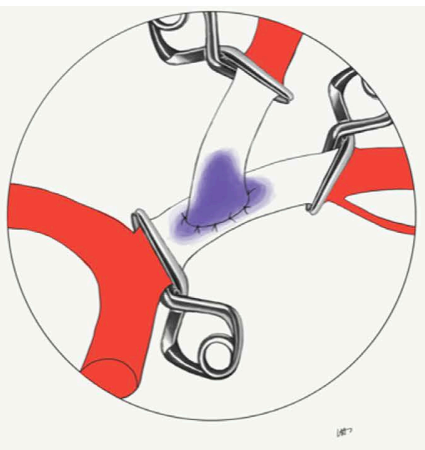

(a)

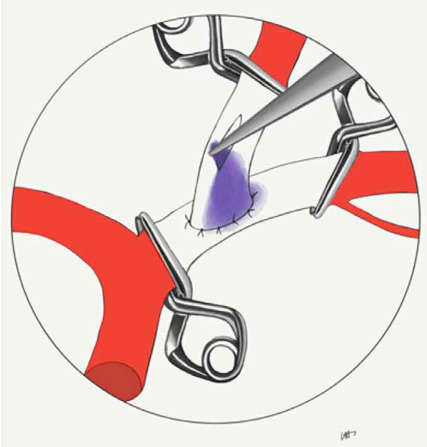

(c)

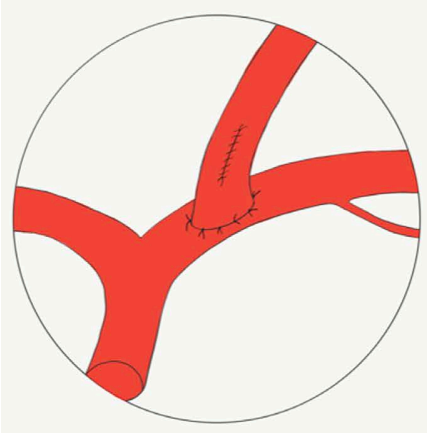

(e)

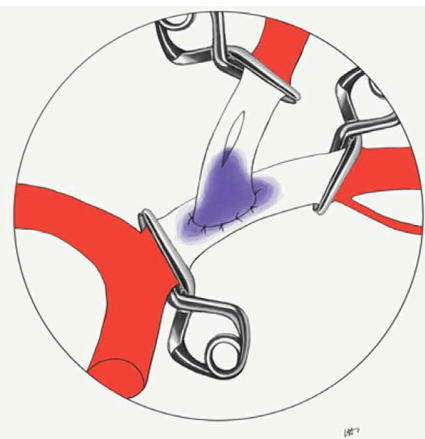

(b)

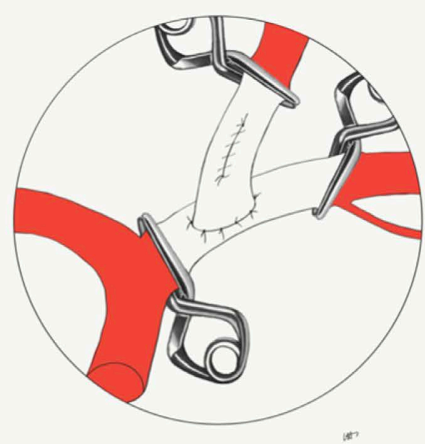

(d)

Figure 5. Thrombectomy through an arteriotomy. 


\subsubsection{Resection of the Anastomosis with Complete Re-Anastomosis}

Complete re-anastomosis in an end-to-side fashion requires the disassembling of the anastomosis after which the thrombus can be extracted (see Figure 6) [9]. The anastomosis can either be redone at the same recipient vessel or, if the anatomy is compatible, a different one. When choosing the anastomotic site, the surgeon must be aware of the available length of the vessel. The anastomosis should preferably not be performed at the site of the previous temporary clips application, because of potential endothelium damage by the vascular clips. If

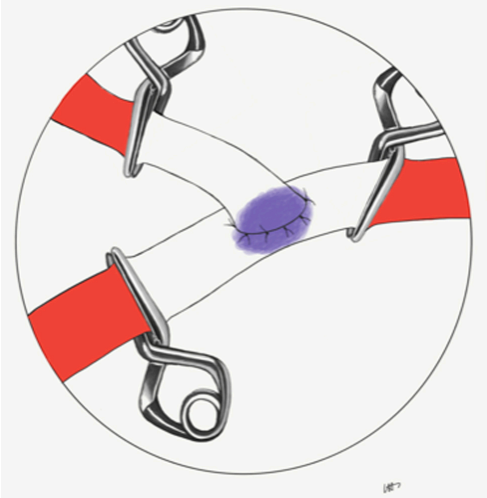

(a)

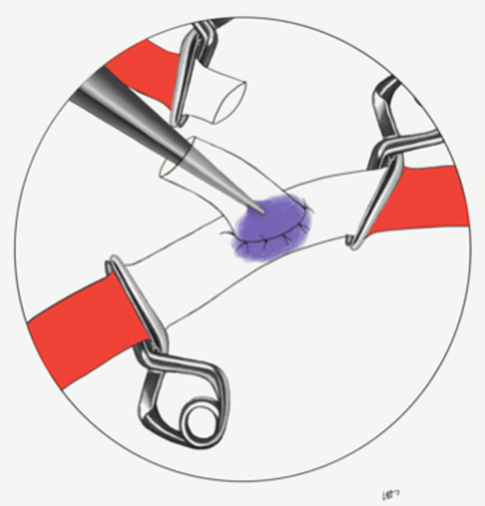

(c)

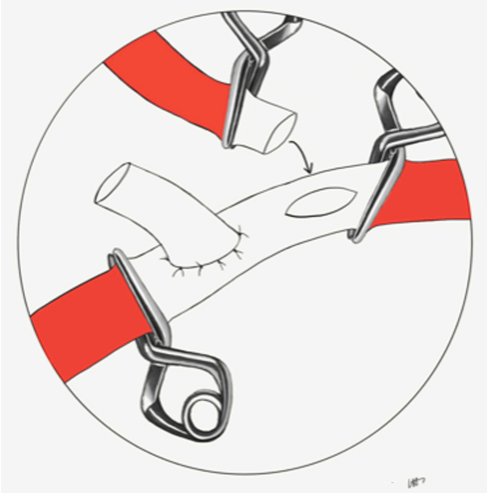

(e)

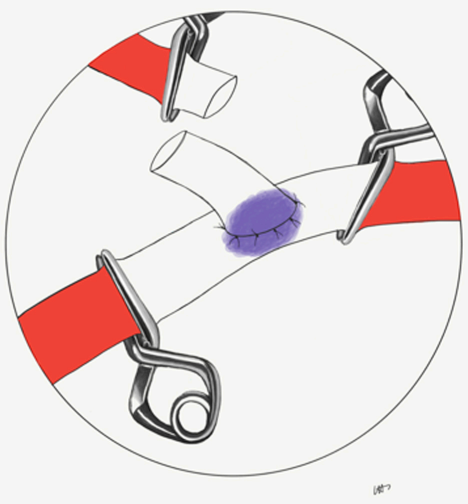

(b)

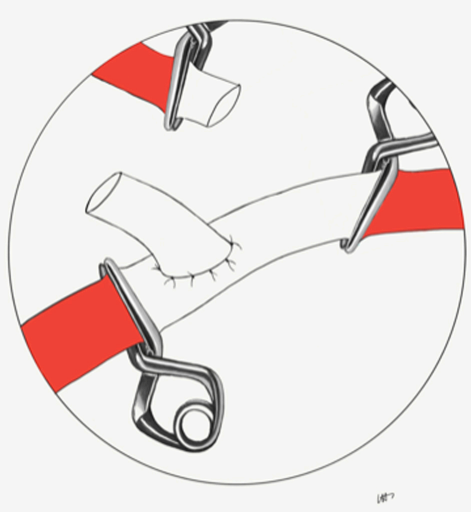

(d)

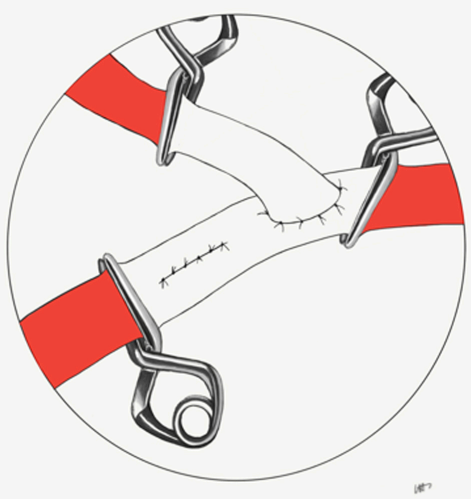

(f)

Figure 6. Complete re-anastomosis end-to-side at a different site. 
there is not enough length of the vessel to be re-anastomosed, interpositional vein graft might be needed.

\subsection{Surgical Salvage Technique: Balloon Extraction}

When the thrombus is not in the direct proximity of the anastomosis, balloon extraction with the fogarthy catheter may be indicated [11]. The technique consists of passing the catheter through the thrombus, gently inflate the balloon and withdraw the catheter including the thrombus. The catheter should be inserted cautiously to avoid intimal irritation. The catheter can be advanced both proximally and distally to the anastomosis. The balloon should not be overinflated and the correct size should be chosen according to the vessel diameter (see Figure 7).

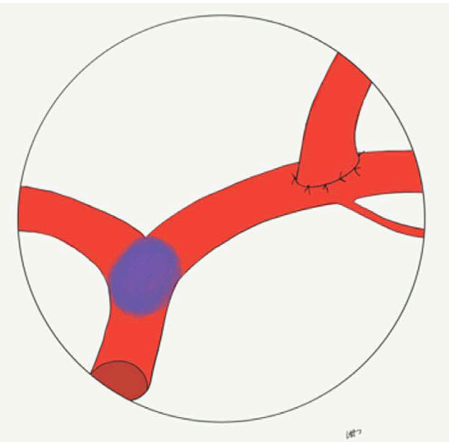

(a)

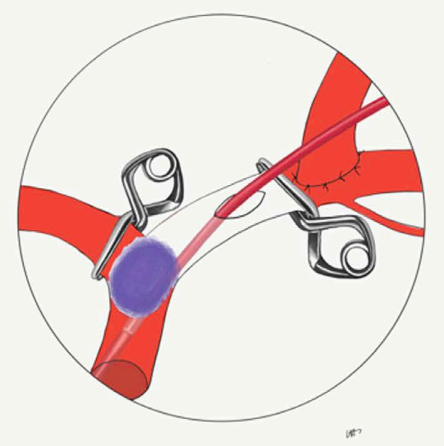

(c)

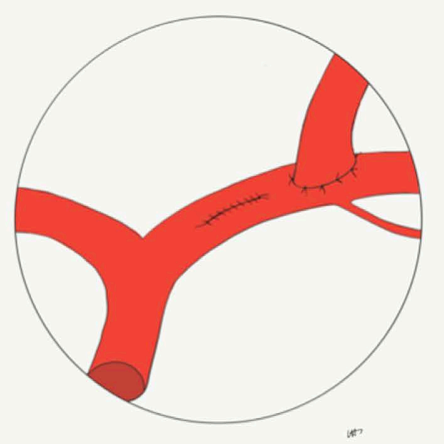

(e)

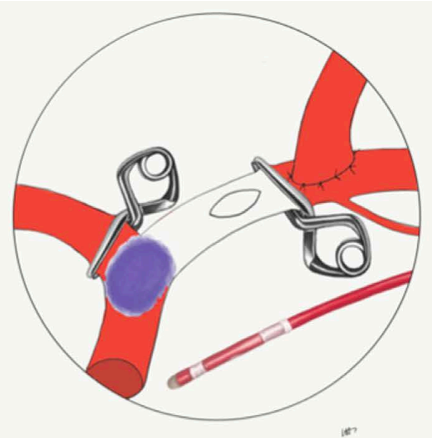

(b)

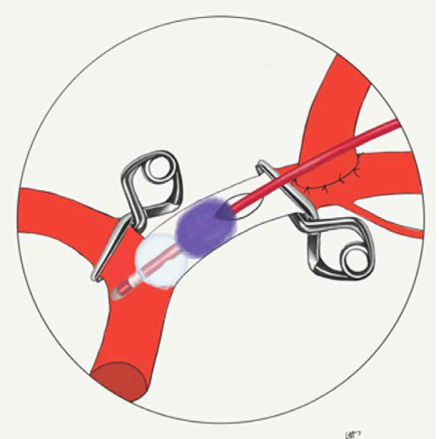

(d)

Figure 7. Balloon extraction. 


\subsection{Anastomosis Occlusion without Thrombus}

A microvascular anastomosis can have inadequate blood flow caused by other means than a thrombus. Altered blood flow can be the result of torsion of the vessel due to excessive traction during the procedure [12]. Torsion can be managed by further dissecting the vessel into the tissue that provides less traction and untwisting will follow automatically (see Figure 8). If the vessel remains twisted despite dissection, re-anastomosis should be performed. Elongation also leads to a decrease in vessel diameter and as with mild torsion, this can be corrected by dissecting into the tissue. Failure of the anastomosis can also be due to an erroneously placed suture passing through the back wall of the vessel. Removal of this suture is necessary. Inadequate blood flow due to pressure of the surrounding tissues must also be ruled out and treated accordingly. Vasospasm can lead to a decrease in blood flow and pharmacological agents such as papaverine have an important role in the management.
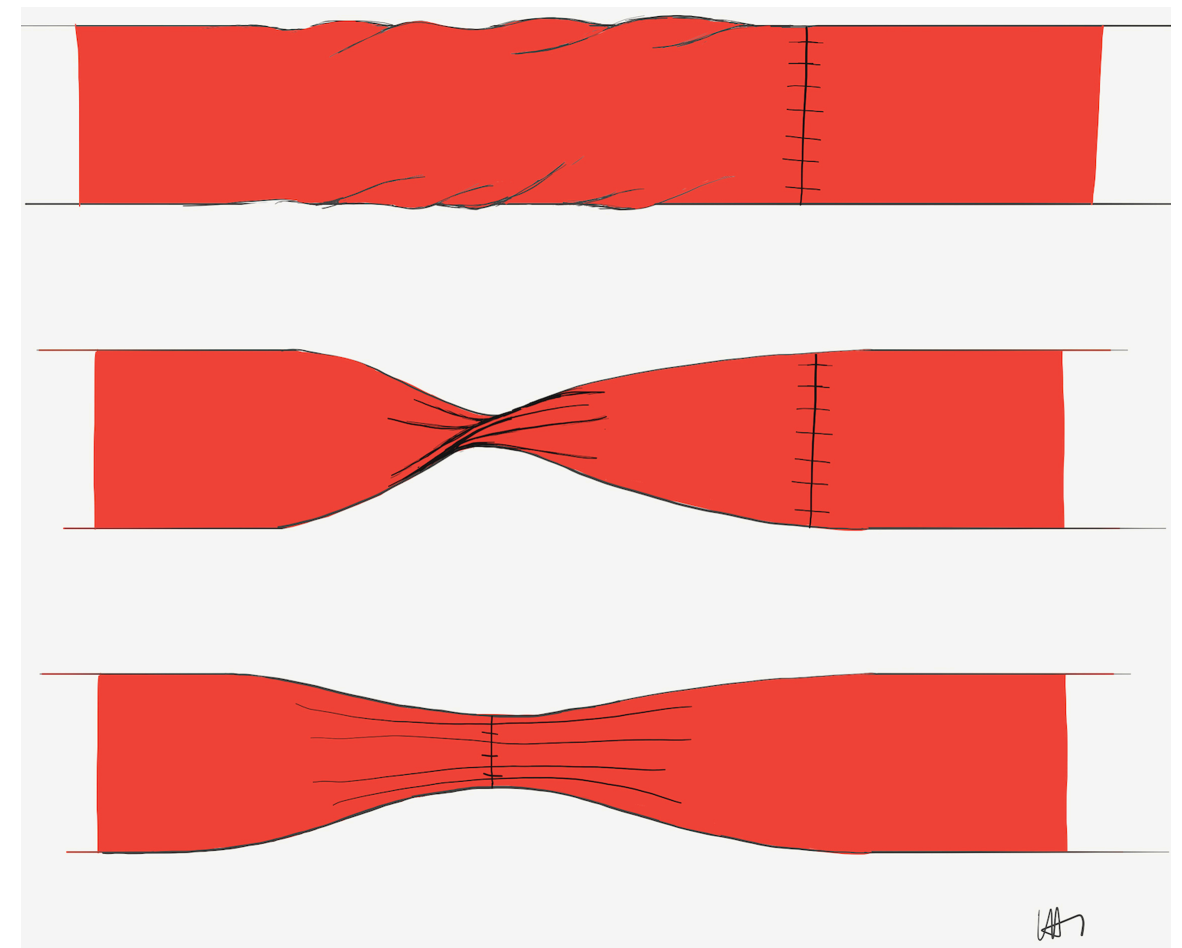

Figure 8. Torsion.

\section{Discussion}

The literature contains not much information and evidence on clinical surgical techniques for patency testing and salvage techniques. Some of the techniques can only be found in textbooks not easily accessible for all. Microvascular surgeons stick to their preferred technique, which they have the most experience with. This paper provides an overview with illustrations and flowcharts to explain possibilities and re-expose hidden knowledge. Before solving the problem, one must be aware of the contributing factors to thrombosis: technical errors 
during microvascular surgery (endothelium manipulation and damage), significant vessel size mismatch, poor vessel quality and smoking [6] [7] [8]. Before microvascular surgery, the patient should at least have stopped smoking for three months.

\subsection{Patency Testing}

Intraoperative assessment of the anastomosis starts with the inspection of the vessel and the pulsation pattern. Flowchart 1 provides an overview of the steps undertaken in clinical patency testing. When removing the temporary clips, a suture-line bleeding can be observed due to needle holes and suture-line holes. This bleeding should stop quickly with the application of gentle pressure. When the blood leakage is managed, the anastomosis becomes clear to inspect the patency. Two types of pulsations can be noticed: longitudinal and expansional. Longitudinal pulsations, where the vessel is lengthened, in combination with a persistent suture-line bleeding are suggestive for a distal occlusion. Expansional pulsations, a circumferential enlargement of the anastomosis, is more indicative for a patent anastomosis. To determine the patency on pulsations pattern only is very hard and therefore, in practice, it should be verified with the flicker or milking test. In small fragile arteries and veins, flicker test is the preferred technique because the milking test is more likely to damage the endothelium and should be retained for the larger arteries in microvascular surgery. If the flicker test cannot clearly confirm a patent anastomosis, the milking test can be used to evaluate the patency.

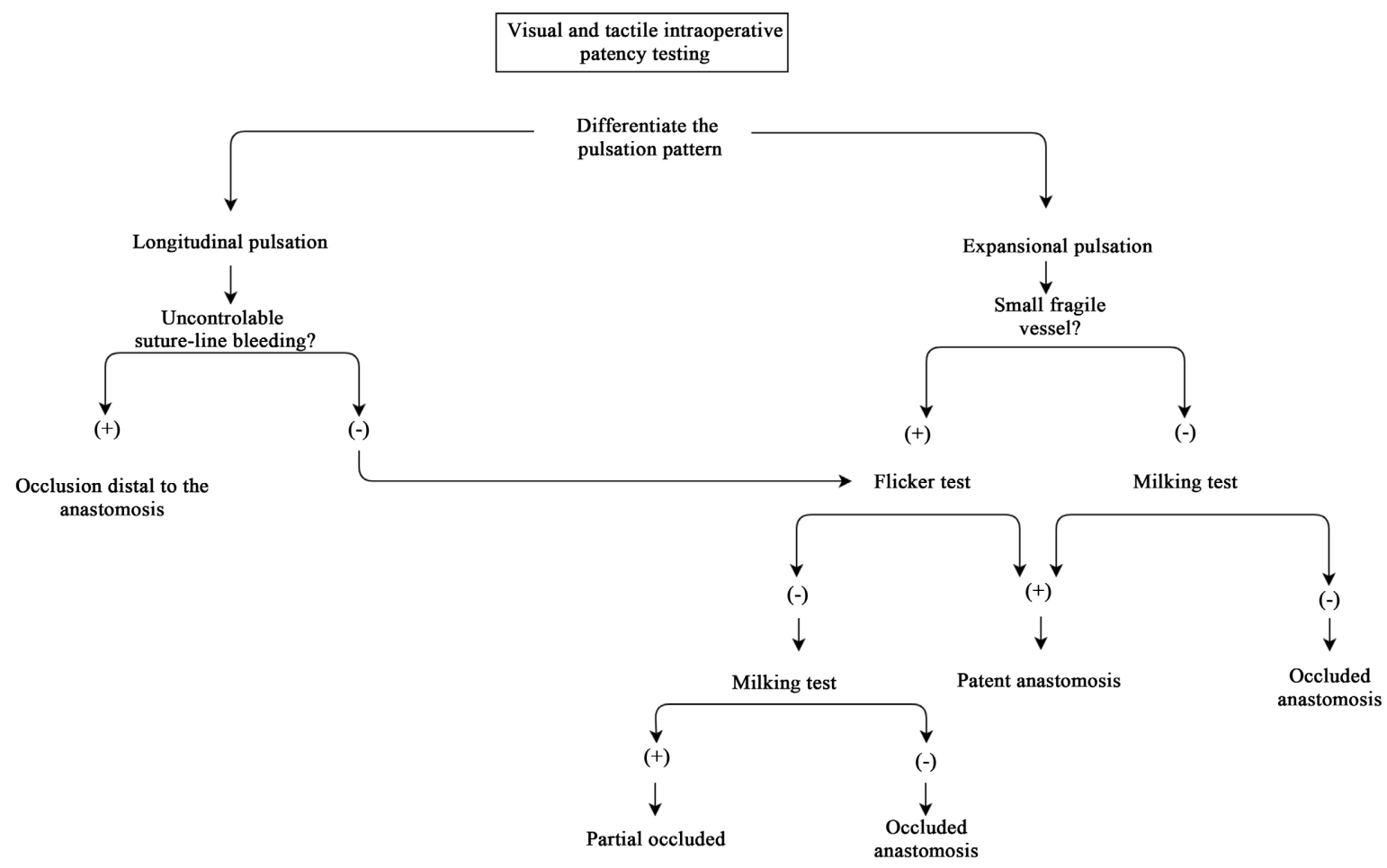

Flowchart 1. Visual and tactile intraoparative patency testing. 


\subsection{Salvage Techniques}

There are two types of thromboses: a white plug and a red plug. Flowchart 2(a) gives a broad overview of which technique to use in what situation. The white plug consists of activated platelets and can easily be crumbled by gently squeezing the vessel wall with microforceps next to the suture line. White plugs can also be disassembled by in situ administration of abciximab, a glycoprotein IIb/IIIa receptor antagonist [13]. This is typically noticed as a white plug being protruded through the suture-line and forms itself mostly within the first 45 minutes after flow restoration [14]. Therefore the first 45 minutes are crucial and frequent intraoperative reassessment of the patency should be done. When the white plug formation remains unnoticed, it will continue to form a red plug. The red plug consists of the formation of a whole thrombus, including activated platelets, coagulation factors and blood cells. Whereas white plugs are platelet-rich and weakly adhesive, red plugs are fibrin-rich and strongly cohesive. The darker in color, the more difficult to disperse a plug will be, requiring some form of disassembly of the anastomosis and extraction of the thrombus [9].

Salvage technique of a red plug depends on the location of the thrombus. When the thrombus is located at the anastomosis, suture-line thrombectomy is the first option for both end-to-end/end-to-side anastomosis. If the thrombus is of significant size or excessive endothelium damage is suspected, anastomotic resection with complete re-anastomosis (either or not with vein graft interposition) is the preferred option to prevent recurrent thrombosis formation. When doing a resection of an end-to-end anastomosis, the thrombus and anastomosis are resected in total, the vessel ends are trimmed and hereafter reconnected. In an end-to-side fashion, the anastomosis is preferably redone using a different site on the recipient vessel because of injured endothelium [9]. Clip damage to the vessel wall is difficult to see, but even seemingly uninjured vessels likely have some degree of endothelial damage and therefore we suggest doing the re-anastomosis at a different site on the same or other recipient vessel. After intraoperative thrombus extraction, each vessel end should be irrigated with a heparin solution in order to prevent recurrent thrombosis. We suggest a heparin solution of $100 \mathrm{U} / \mathrm{ml}$. Thrombectomy through a longitudinal arteriotomy is a valid option if the anastomosis is faultless with little endothelium damage and the small thrombus is located nearby the anastomosis. If the thrombus is located away from the anastomosis and beyond the surgeons reach, a balloon extraction with Fogarthy catheter is the choice of method. In the context of prevention, manipulation and damage of the endothelium increase the risk of developing an intraoperative thrombosis. Therefore, being very cautious with the endothelium throughout the whole microsurgical procedure is of utter importance.

\subsection{No Thrombus Occlusion}

A microvascular anastomosis can have inadequate blood flow caused by other means than a thrombus, see Flowchart 2(b). An erroneously placed stitch 


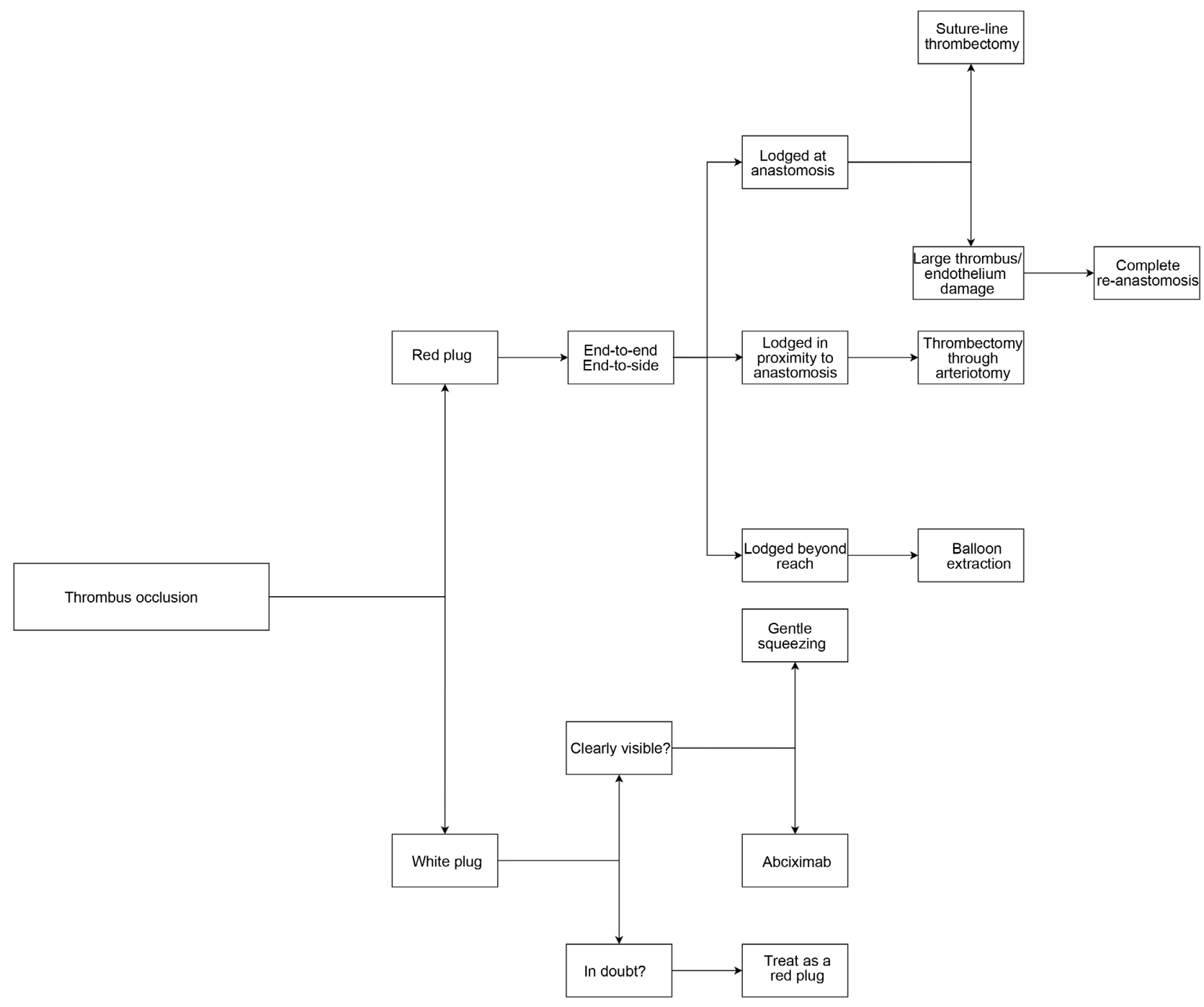

(a)

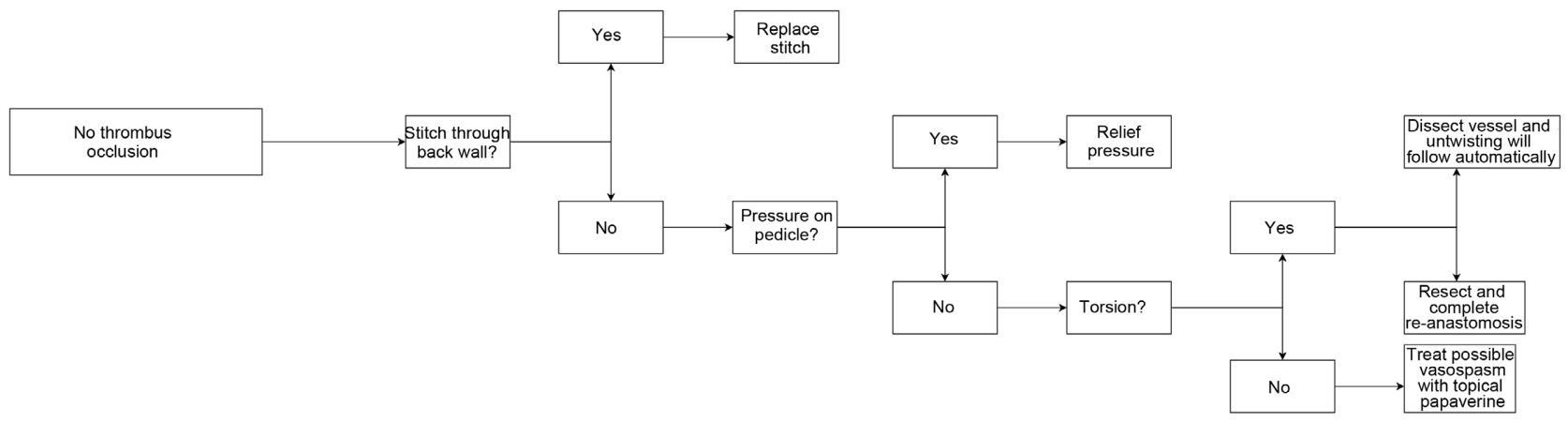

(b)

Flowchart 2. (a) Thrombus occlusion salvage techniques; (b) No thrombus occlusion salvage techniques.

through the back wall of the vessel will cause a diminution in blood flow and is also thrombogenic. The misplaced stitch should be replaced accordingly. With the replacement of the stitch, inspection of the intima must be done to rule out intimal tear. Pressure on the pedicle can cause insufficient blood flow and the 
pressure should be relieved immediately. Torsion or elongation of the vessel due to excessive traction is treated either by dissection into the tissue or resection with complete re-anastomosis. Vasospasm can also be the cause of altered flow and is treated with pharmacological agents.

\section{Conclusion}

This article includes most of the basic surgical techniques for micro-anastomosis patency testing and intraoperative thrombosis salvage. To check the patency of a microvascular anastomosis, the pulsation pattern, flicker test and milking test can be used. The surgical salvage of an intraoperative thrombosis depends on the localization of the thrombus and one can rely on suture-line thrombectomy, thrombectomy through an arteriotomy, complete re-anastomosis and a balloon catheter. In case of any doubt, it is better to reopen a few sutures and have a clear inspection of the anastomosis in order to prevent redo surgeries. The comprehensive illustrations and flowcharts give a good overview and are especially convenient for the starting microsurgeon to increase the knowledge on how to handle this significant complication.

\section{Acknowledgements}

We would like to thank graphic designer Ms. Lemieh Hajjab for designing the illustrations for this paper.

\section{Conflicts of Interest}

The authors declare no conflicts of interest regarding the publication of this paper.

\section{References}

[1] Schusterman, M.A., Miller, M.J., Reece, G.P., Kroll, S.S., Marchi, M. and Goepfert, H. (1994) A Single Center's Experience with 308 Free Flaps for Repair of Head and Neck Cancer Defects. Plastic and Reconstructive Surgery, 93, 472-478; Discussion 9-80. https://doi.org/10.1097/00006534-199403000-00004

[2] Urken, M.L., Weinberg, H., Buchbinder, D., Moscoso, J.F., Lawson, W., Catalano, P.J., et al. (1994) Microvascular Free Flaps in Head and Neck Reconstruction. Report of 200 Cases and Review of Complications. Archives of Otolaryngology-Head \& Neck Surgery, 120, 633-640. https://doi.org/10.1001/archotol.1994.01880300047007

[3] Tsai, T.M., Bennett, D.L., Pederson, W.C. and Matiko, J. (1988) Complications and Vascular Salvage of Free-Tissue Transfers to the Extremities. Plastic and Reconstructive Surgery, 82, 1022-1026. https://doi.org/10.1097/00006534-198812000-00013

[4] Harashina, T. (1988) Analysis of 200 Free Flaps. British Journal of Plastic Surgery, 41, 33-36. https://doi.org/10.1016/0007-1226(88)90141-5

[5] Irons, G.B., Wood, M.B. and Schmitt 3rd, E.H. (1987) Experience with One Hundred Consecutive Free Flaps. Annals of Plastic Surgery, 18, 17-23. https://doi.org/10.1097/00000637-198701000-00005 
[6] Couteau, C., Rem, K., Guillier, D., Moris, V., Revol, M. and Cristofari, S. (2018) Improving Free-Flap Survival Using Intra-Operative Heparin: Ritualistic practice or Evidence-Base Medicine? A Systematic Review. Annales de Chirurgie Plastique et Esthetique, 63, e1-e5. https://doi.org/10.1016/j.anplas.2017.06.010

[7] Hanasono, M.M. and Butler, C.E. (2008) Prevention and Treatment of Thrombosis in Microvascular Surgery. Journal of Reconstructive Microsurgery, 24, 305-314.

https://doi.org/10.1055/s-2008-1080530

[8] O’Neill, A.C., Haykal, S., Bagher, S., Zhong, T. and Hofer, S. (2016) Predictors and Consequences of Intraoperative Microvascular Problems in Autologous Breast Reconstruction. Journal of Plastic, Reconstructive \& Aesthetic Surgery. JPRAS, 69, 1349-1355. https://doi.org/10.1016/j.bjps.2016.07.006

[9] Lawton, M.T. (2018) Seven Bypasses: Tenets and Techniques for Revascularization. Thieme Medical Publishers, Inc., New York, 150-162.

https://doi.org/10.1055/b-006-160171

[10] Ichinose, A., Tahara, S., Terashi, H., Nomura, T. and Omori, M. (2003) Short-Term Postoperative Flow Changes after Free Radial Forearm Flap Transfer: Possible Cause of Vascular Occlusion. Annals of Plastic Surgery, 50, 160-164. //doi.org/10.1097/01.SAP.0000037264.92535.AC

[11] Egozi, D., Fodor, L. and Ullmann, Y. (2011) Salvage of Compromised Free Flaps in Trauma Cases with Combined Modalities. Microsurgery, 31, 109-115. https://doi.org/10.1002/micr.20852

[12] Yu, J., Qu, L., Xu, B., Wang, S., Li, C., Xu, X., et al. (2017) Current Understanding of Dolichoarteriopathies of the Internal Carotid Artery: A Review. International Journal of Medical Sciences, 14, 772-784. https://doi.org/10.7150/ijms.19229

[13] Buchanan, I.A., Lee, B., Amar, A.P. and Giannotta, S.L. (2018) In Situ Administration of Abciximab for Thrombus Resolution during Intracranial Bypass Surgery: Case Report. Journal of Neurosurgery, 130, 268-272. https://doi.org/10.3171/2017.8.JNS17430

[14] Tomasello, F., Spadaro, A., Albanese, V. and Conforti, P. (1981) SEM Evaluation of Endothelial Changes Following End-to-Side Microvascular Anastomoses in Rats. Journal of Neurosurgery, 54, 525-531. https://doi.org/10.3171/jns.1981.54.4.0525 